\title{
MAGNITUDE OF VANCOMYCIN RESISTANT ENTEROCOCCUS FECAL COLONIZATION AND BACTEREMIA IN PATIENTS WITH HEMATOLOGICAL DISEASES AT TERTIARY BONE MARROW TRANSPLANT CENTRE RAWALPINDI
}

\author{
Nargis Sabir, Luqman Satti*, Abeera Ahmed**, Gohar Zaman***, Raheel Iftikhar***, Nasir Uddin***** \\ Combined Military Hospital Lahore/National University of Medical Sciences (NUMS) Pakistan, *Pakistan Naval Ship Shifa Hospital, Karachi Pakistan, \\ ${ }^{* *}$ Combined Military Hospital Malir/National University of Medical Sciences (NUMS) Pakistan, ${ }^{* * *}$ Armed Forces Institute of Pathology/National University of \\ Medical Sciences (NUMS) Rawalpindi Pakistan, ${ }^{* * * *}$ Armed Forces Bone Marrow Transplant Center/National University of Medical Sciences (NUMS) Rawalpindi \\ Pakistan, ${ }^{* * * *}$ Army Medical College/National University of Medical Sciences (NUMS) Rawalpindi Pakistan
}

\section{ABSTRACT}

Objective: To discover the frequency of vancomycin resistant enterococci (VRE) fecal colonization and subsequent bacteremia in patients with hematological diseases in a bone marrow transplant center.

Study Design: Cross-sectional study.

Place and Duration of Study: Department of Microbiology, Armed Forces Institute of Pathology (AFIP), in collaboration with Armed Forces Bone Marrow Transplant Center, Rawalpindi, from Jan 2016 to Dec 2019.

Methodology: Stool specimens/anal swabs from all enrolled patients were collected aseptically and transported to the laboratory without delay. Blood cultures were collected aseptically from only those enrolled patients who developed signs and symptoms of bacteremia. Stool and blood cultures were processed as per standard microbiological protocols. Enterococci were identified to species level by colony morphology and biochemical tests. Modified Kirby Bauer disc diffusion method and VITEK-2 system (Version-8.01 bio Merieux, France) were used to perform antimicrobial sensitivity of each isolate.

Results: A total number of 246 patients were studied. Among them, $67(27 \%)$ patients had fecal colonization by vancomycin resistant enterococci. We report a statistically significant association of recent hospitalization, prolonged exposure to antimicrobial therapy, chemotherapy exposure and use of indwelling devices during the hospital stay with vancomycin resistant enterococci colonization. Vancomycin resistant enterococci bacteremia was detected in 57 (23\%) patients. Among these 57 patients, $53(93 \%)$ were vancomycin resistant enterococci colonizers. Vancomycin resistant enterococci colonization was significantly associated with vancomycin resistant enterococci bacteremia.

Conclusion: A considerable burden of vancomycin resistant enterococci fecal colonization exists among patients with hematological diseases. vancomycin resistant enterococci colonization poses a considerable risk of vancomycin resistant enterococci bloodstream infections in this patient population.

Keywords: Antimicrobial susceptibility, Hematological diseases, Vancomycin resistant enterococci bacteremia, Vancomycin resistant enterococci colonization.

This is an Open Access article distributed under the terms of the Creative Commons Attribution License (https://creativecommons.org/licenses/by-nc/4.0/), which permits unrestricted use, distribution, and reproduction in any medium, provided the original work is properly cited.

\section{INTRODUCTION}

For several countries around the world, healthcare associated infections with some pathogenic bacteria has risen exponentially. Resistance to antimicrobials has posed a severe threat to treating clinicians and health care authorities ${ }^{1}$. Enterococci are an essential component of lower gastrointestinal tract bacterial flora. They are low virulent pathogen and usually cause opportunistic infections. Enterococci are one of predominant health care associated pathogens in the United States with $29 \%$ of vancomycin resistance 2 . Vancomycin-resistant Enterococci (VRE) are important health care associated pathogen that commonly affect seriously ill patients. Remarkable genetic plasticity of VRE allow these pathogens to acquire antimicrobial resistance gene easily ${ }^{3}$. Since their emergence in 1980s,

Correspondence: Dr Nargis Sabir, House No. 134, Street 2, Sector-B Askari 10, Lahore Pakistan

Received: 09 Jul 2020; revised received: 28 Oct 2020; accepted: 17 Nov 2020 there has been a continuous expansion in the prevalence of VRE across the globe ${ }^{4}$. Gastrointestinal (GI) tract colonization is common and bacteremia with VRE has become a noteworthy complication in patients with hematological disorders ${ }^{5}$.

The evolution of VRE as a significant healthcareassociated pathogen is attributable to its ability to colonize the gastrointestinal (GI) tract, tenacity in the healthcare setting, genomic plasticity, mobile genetic parts and increased fatality ${ }^{6}$. GI tract is the most common site of VRE colonization. This colonization can persist for months to years 7 . Additionally, VRE are also substantial nosocomial pathogens in patients with hematological malignancies ${ }^{8,9}$.

Data on the scale of VRE colonization, its antimicrobial susceptibility patterns, correlated factors and subsequent development of VRE bacteremia has not been well studied in our country. Therefore, this study was planned to establish the burden of VRE coloniza- 
tion and subsequent bacteremia in patients with hematological diseases in a bone marrow transplant center.

\section{METHODOLOGY}

This was a cross-sectional study using non probability consecutive sampling technique conducted in department of Microbiology, Armed Forces Institute of Pathology (AFIP), in collaboration with Armed Forces Bone Marrow Transplant Center, Rawalpindi, from January 2016 to December 2019. Study population included all the patients (indoor and outdoor) irrespective of age and gender suffering from any hematological disorder. These patients were enrolled in this study after taking informed consent. Repeated stool specimens/anal swabs of enrolled patients were excluded from the study. Permission was sought from institutional ethics committee (FC-MIC-14-5/READ-IRB/397). Demographics and various risk factors of enrolled patients like age, gender, disease, recent hospitalization, recent prolonged exposure to antimicrobials, exposure to chemotherapy, and use of indwelling devices during hospital stay were recorded in the especially designed preforma.

Recent hospitalization was defined as admission of any duration in any health care setting in previous one month. Recent prolonged exposure to antimicrobials was defined as administration of any antimicrobial for more than two weeks in preceding one month. VRE colonization was described as presence of VRE isolates in stool/anal swabs of patients without causing any symptoms or disease. Sample size was assessed using WHO sample size calculator, using specified absolute precision of $0.05,95 \%$ confidence level and 0.2010 anticipated population proportion. Estimated sample size was 246 .

Stool specimens/anal swabs from all enrolled patients were collected aseptically and transported to laboratory without delay. Blood cultures were collected aseptically from only those enrolled patients who developed signs and symptoms of bacteremia. The stool specimens/anal swabs were cultured on Chrom ID VRE agar (bioMerieux, France) and incubated at $37^{\circ} \mathrm{C}$ in ambient air for $24-48 \mathrm{hrs}$. Blue-green and violet coloured colonies were provisionally identified as VRE. Positive blood cultures and coloured colonies on selective agar were then subcultured to blood agar (Oxoid CM 0055, UK) and incubated at $37^{\circ} \mathrm{C}$ in ambient air for $24 \mathrm{hrs}$. For identification at genus level, colony morphology, Gram staining, catalase reaction, growth in $6.5 \% \mathrm{NaCl}$, bile esculin hydrolysis and 1\% arabinose fermentation were used. Moreover, to detect
Enterococci at species levels, API20 STREP (bio Merieux, France) strips were used.

Standard modified Kirby Bauer disc diffusion method and VITEK 2 systems-Version-8.01(bio Merieux, France) were used to perform antimicrobial susceptibility testing. Clinical Laboratory Standards Institute (CLSI) guidelines ${ }^{11}$, were applied for interpreting disks zone and MICs. Antimicrobial susceptibility test was performed against antibiotics such as; vancomycin $(30 \mu \mathrm{g})$, teicoplanin $(30 \mu \mathrm{g})$, penicillin (10units), ampicillin $(10 \mu \mathrm{g})$, linezolid $(30 \mu \mathrm{g})$ and doxycycline $(30 \mu \mathrm{g})$ (Oxoid, UK). Strict quality control was ensured by preparing culture media according to the instructions given by manufacturer. The sterility of culture media batch was ensured by incubating the plate at $37^{\circ} \mathrm{C}$ for 24 h. E. faecalis ATCC 29212 and E. coli ATCC 25922 standard strains were utilized as control strains.

Positive cultures for VRE were reported as "VRE colonization is detected. No antibiotic therapy is recommended to treat colonization." The antibiogram of all the positive cultures were saved and reports delivered on clinician request. This antibiogram was also used to guide empirical therapy when colonized patients developed bacteremia.

When VRE was isolated, hospital staff/concerned clinician was given an alert call to take strict infection control measures to limit its nosocomial spread. Standard and contact based isolation precautions were implemented for all VRE colonized/infected patients.

All findings were assessed using SPSS-23 version. Descriptive statistics were used for both qualitative and quantitative variables. For qualitative variables like gender, disease, VRE colonization, antibiotic susceptibility and VRE bacteremia frequency and percentages were calculated. For quantitative variables like age mean \pm SD was calculated. To examine the effect of modifiers on outcome, stratification and poststratification Fisher's Exact test were applied for categorical data. Two-tailed $p$-values $\leq 0.05$ were considered significant.

\section{RESULTS}

Overall 246 patients including 173 (70\%) males and $73(30 \%)$ females were studied. Average age of the patients was $20 \pm 14$ years. Clinical diagnosis of the enrolled patients was; acute myeloid leukemia 64 $(26 \%)$ beta thalassemia major $54(22 \%)$, aplastic anemia $42(17 \%)$, chronic myeloid leukemia $26(11 \%)$, acute lymphoblastic leukemia 24 (9.5\%), hemophagocytic lymphohistiocytosis 14 (5.7\%) and paroxysmal nocturnal 
hemoglobinuria 12 (4.5\%). Patients suffering from hematological disorders other than mentioned above were $10(4 \%)$.

VRE fecal colonization was detected in $67(27 \%)$ of patients. Among these 67 VRE colonizers, 63 (94\%) and $4(6 \%)$ were colonized by Enterococcus faecium and Enterococcus faecalis respectively. Association of recent hospitalization, recent prolonged exposure to antimicrobials, exposure to chemotherapy and use of indwelling devices during hospital with VRE colonization is shown in table-I. $p$-value $<0.05$ was measured as substantial. The two-tailed $p$-value $<0.0001$ suggested that relationship between rows (groups) and columns (outcomes) was statistically significant.

Antibiotic susceptibility of VRE isolates cultured from stool specimens/anal swabs showed that in addition to vancomycin resistance these isolates were resistant to penicillin $(100 \%)$, ampicillin $(100 \%)$, doxycycline $(88 \%)$ and teicoplanin $(73 \%)$. All VRE isolates were sensitive to linezolid.

VRE bacteremia was detected in $57(23 \%)$ patients. Among these 57 patients 53 (93\%) were VRE colonizers. Association of VRE colonization with VRE bacteremia was determined using Fisher exact test and shown in the table-II. $p$-value $<0.05$ was considered as significant. The two-tailed $p$-value $<0.0001$ revealed that the relationship between rows (groups) and columns (outcomes) was statistically significant. All VRE

Table-I: Association of vancomycin resistant enterococcus (VRE) colonization with various risk factors $(n=246)$.

\begin{tabular}{|c|c|c|c|c|}
\hline & \multicolumn{2}{|c|}{ VRE Colonization } & \multirow[b]{2}{*}{$\begin{array}{c}p \text { - } \\
\text { value }\end{array}$} \\
\hline & & $\begin{array}{c}\text { Colonizers } \\
67(27 \%)\end{array}$ & $\begin{array}{c}\text { Non } \\
\text { Colonizers } \\
179(73 \%)\end{array}$ & \\
\hline \multirow{2}{*}{$\begin{array}{l}\text { Recent } \\
\text { Hospitalization }\end{array}$} & Yes & $66(99 \%)$ & $100(56 \%)$ & \multirow{8}{*}{$<0.001$} \\
\hline & No & $1(1 \%)$ & $79(44 \%)$ & \\
\hline \multirow{2}{*}{$\begin{array}{l}\text { Prolonged } \\
\text { Exposure to } \\
\text { antimicrobials }\end{array}$} & Yes & $66(99 \%)$ & $119(66 \%)$ & \\
\hline & No & $1(1 \%)$ & $60(34 \%)$ & \\
\hline \multirow{2}{*}{$\begin{array}{l}\text { Chemotherapy } \\
\text { exposure }\end{array}$} & Yes & $65(97 \%)$ & $66(37 \%)$ & \\
\hline & No & $2(3 \%)$ & $113(63 \%)$ & \\
\hline \multirow{2}{*}{$\begin{array}{l}\text { Indwelling } \\
\text { Devices }\end{array}$} & Yes & $65(97 \%)$ & $56(31 \%)$ & \\
\hline & No & $2(3 \%)$ & $123(69 \%)$ & \\
\hline
\end{tabular}

Table-II: Association of vancomycin resistant enterococcus (VRE) colonization with VRE bacteremia.

\begin{tabular}{|c|c|c|c|c|}
\hline & \multicolumn{2}{|c|}{ VRE Bacteremia } & \multirow[b]{2}{*}{$\begin{array}{c}p- \\
\text { value }\end{array}$} \\
\hline & & $\begin{array}{c}\text { Yes } \\
57(23 \%)\end{array}$ & $\begin{array}{c}\text { No } \\
189(77 \%)\end{array}$ & \\
\hline \multirow{2}{*}{$\begin{array}{l}\text { VRE Fecal } \\
\text { Colonization }\end{array}$} & Colonizers & $53(79 \%)$ & $14(21 \%)$ & \multirow[b]{2}{*}{$<0.001$} \\
\hline & $\begin{array}{c}\text { Non } \\
\text { Colonizers }\end{array}$ & $4(2 \%)$ & $175(98 \%)$ & \\
\hline
\end{tabular}

isolates recovered from blood cultures of colonized patients who developed sepsis had similar antibiogram as that of colonizer.

\section{DISCUSSION}

The global spread of VRE and other MDROs (multidrug resistant organisms) complicates treat-ment and isolation measures in hospital ${ }^{12}$. Various risk factors for VRE colonization include past contact to multiple antibiotics, previous hospitalization, close contact with VRE-colonized patients, immune suppression, neutropenia and renal insufficiency ${ }^{13}$. The ability of Enterococci spp to colonize the human gastrointestinal tract and their prolonged survival on environmental surfaces increase their ability to cause health care associated (HAI) infections ${ }^{14}$. The typical pathway of acquiring nosocomial VRE begins with the direct contact with contaminated hands or objects. Gut microbiota is then suppressed through anti-microbial selective pressure, ratifying for colonization of VRE, as it is inherently resistant to several antibiotics. When the patient becomes immunocompromised, VRE multiplies and trigger a clinical illness ${ }^{15}$. Bloodstream infections caused by VRE are a major cause of morbidity and death in patients with solid and hematologic malignancies and are usually preceded by GI tract VRE colonization ${ }^{16}$. In patients with malignancy, mucositis caused by chemotherapy allows bacterial translo-cation from GI tract into the bloodstream ${ }^{17}$. Rapid and accurate identification of VRE is critical not only in management and treatment of VRE infections but also for the prevention of its nosocomial spread ${ }^{13}$. Little information is available about the prevalence of VRE fecal colonization and subseq-uent bacteremia in Pakistan, especially among hematological diseases patients.

In our study, the overall frequency of VRE colonization in patients with hematological disorders was $27 \%$ which was comparable with $24 \%$ reported by meta-analysis done by Alevizakos et $a l^{10}$. Another study conducted by Akturk et al, in Pediatric hematology-oncology department showed 31.4\% VRE fecal colonization which was comparatively high as compared to our study ${ }^{18}$.

In our study, Enterococcus faecium were the most frequent isolated species ( $94 \%$ of isolates), followed by Enterococcus faecalis (6\% of isolates). Related studies conducted by Matar et al and Rolston et al also reported Enterococcus faecium as most prevalent isolate among VRE colonized cancer patients ${ }^{19,20}$. 
A significant association of VRE colonization with recent hospitalization, prolong exposure to antimicrobial therapy, chemotherapy exposure and use of indwelling devices during hospital stay was found in this study. In line to our study, other study conducted by Suntharam et al, Batistao et al, and Agegne et al, also revealed significant association of previous prolonged intake of antibiotics, previous history of hospitalization with VRE colonization 5,16,21. Sakka et al. Performed a case control study in which utilization of invasive devices and extended exposure to antimicrobials pre-dicted VRE colonization in multivariate analysis. These findings were also concordant with our study ${ }^{22}$. However, a unique finding of our study is substantial association of VRE colonization with chemotherapy exposure. Bacteremia by VRE was found in $23 \%$ of patients. In contrast to our study, lower prevalence of VRE bacteremia $(1.5 \%)$ was described in another study conducted by Kara $e^{2} a^{23}$. A possible explanation for high prevalence of VRE bacteremia in our study was exclusive inclusion of patients with hematological disorders.

VRE colonization significantly escalates the risk for successive VRE bloodstream infection. A statistically significant association of VRE colonization with subsequent VRE bacteremia was found in our study. Our study findings were consistent with other studies conducted by Alevizakos et al ${ }^{10}$ and Ozsoy et al ${ }^{13}$.

Adherence to antimicrobial stewardship, comprehensive testing and ongoing surveillance of VRE colonization and infections are the need of hour to curb the nosocomial spread of this superbug 24 .

\section{RECOMMENDATIONS}

1. Rigorous infection control procedures must be emphasized for VRE colonizers to limit nosocomial spread of infection.

2. VRE fecal screening must be done in hematological diseases patients with recent hospitalization, prolong exposure to antimicrobial therapy, chemotherapy exposure and use of indwelling devices during hospital stay.

3. VRE colonizers must be closely monitored for VRE infections especially bacteremia. If such patients developed bacteremia then empirical antimicrobial therapy must be instituted according to colonizer antibiogram.

\section{ACKNOWLEDGEMENT}

The study has been sponsored by Armed Forces Institute of Pathology Rawalpindi Pakistan. Daud
Yahya B.E (Civil Engineer and Statistician) has done statistical analysis.

\section{CONCLUSION}

A considerable VRE colonization problem exists among patients with hematological diseases. Patients with recent hospitalization, prolong exposure to antimicrobial therapy, chemotherapy exposure and use of indwelling devices during hospital stay are particularly at high risk of developing VRE colonization. VRE colonization poses a considerable risk of VRE blood stream infections in this patient population. However, more studies on large scale with and more diverse patient population should be carried out to know the true burden of VRE colonization and subsequent bacteremia.

\section{CONFLICT OF INTEREST}

This study has no conflict of interest to be declared by any author.

\section{REFERENCES}

1. Elstrom P, Astrup E, Hegstad K, Samuelsen O, Enger H, Kacelnik $\mathrm{O}$. The fight to keep resistance at bay, epidemiology of carbapenemase producing organisms (CPOs), vancomycin resistant enterococci (VRE) and methicillin resistant Staphylococcus aureus (MRSA) in Norway, 2006-2017. PLos One 2019; 14(2): e021174145.

2. Hidron AI, Edwards JR, Patel J, Horan TC, Sievert DM, Pollock DA et al. NHSN annual update: Antimicrobial-resistant pathogens associated with healthcare-associated infections: Annual summary of data reported to the National Healthcare Safety Network at the Centers for Disease Control and Prevention, 20062007. Infect Control Hosp Epidemiol 2008; 29(11): 996-11.

3. Contreras GA, Munita JM, Arias CA. Novel Strategies for the Management of Vancomycin-Resistant Enterococcal Infection. Curr Infect Dis Rep 2019; 21(7): 22-26.

4. Weiner LM, Webb AK, Limbago B, Patel J, Kallen AJ, Edwards JR. et.al. Antimicrobial-resistant pathogens associated with healthcare-associated infections: summary of data reported to the national healthcare safety network at the centers for disease control and prevention, 2011-2014. Infect Control Hosp Epidemiol 2016; 37(11): 1288-301.

5. Suntharam N, Lankford MG, Trick WE and Peterson L. Risk factors of acquisition of vancomycin-resistant enterococci among hematology-oncology patients. Diagnostic Microbiol Infect Dis 2002; 43(3): 183-88.

6. Weiss A, Domig K, Kneifel W. Comparison of selective media for the enumeration of probiotic enterococci from animal feed. Food Technol Biotechnol 2005; 43(2): 147-55.

7. Baden LR, Critchley IA, Sahm DF, So W, Gedde M, Porter S. et.al. Molecular characterization of vancomycin- resistant enterococci repopulating the gastrointestinal tract following treatment with a novel glycolipodepsipeptide, ramoplanin. J Clin Microbiol 2002; 40(4): 1160-63.

8. Worth LJ, Thursky KA, Seymour JF, Slavin MA. Vancomycinresistant Enterococcus faecium infection in patients with hematologic malignancy: patients with acute myeloid leukemia are at high risk. Eur J Haematol 2007; 79(3): 226-233. 
9. Mac S, Fitzpatrick T, Johnstone J, Sander B. Vancomycin-resistant enterococci (VRE) screening and isolation in the general medicine ward: a cost-effectiveness analysis. Antimicrob Resist Infect Control 2019; 8(1): 168-72.

10. Alevizakos M, Gaitanidis A, NasioudisD, Tori K, Flokas ME, Mylonakis E. Colonization with vancomycin-resistant enterococci and risk for bloodstream infection among patients with malignancy: a systematic review and meta-analysis. Open Forum Infect Dis 2017; 4(1): 246-49.

11. Clinical and Laboratory Standards Institute (CLSI). Performance standards for antimicrobial susceptibility testing; Twenty fourth informational supplement M100-S24. Wayne, A: CLSI; 2019. [Internet] Available at: https://www.nih.org.pk/wp-content/ uploads/2021/02/CLSI-2020.pdf.

12. Ballo O, Tarazzit I, Stratmann J, Reinheimer C, Hogardt M, Wichelhaus TA, et. al. Colonization with multidrug resistant organisms determines the clinical course of patients with acute myeloid leukemia undergoing intensive induction chemotherapy. PLoS One 2019; 14(1): e0210991-95.

13. Ozsoy S, Llki A. Detection of vancomycin-resistant enterococci (VRE) in stool specimens submitted for Clostridium difficile toxin testing. Braz J Microbiol 2017; 48(3): 489-92.

14. MacDougall C, Johnstone J, Prematunge C, Adomako K, Nadolny E, Truong E et al. Economic evaluation of vancomycinresistant enterococci (VRE) control practices: a systematic review. J Hosp Infect 2020; 105(1): 53-63.

15. Linden PK. Optimizing therapy for vancomycin-resistant enterococci (VRE). Semin Respir Crit Care Med 2007; 28(6): 632-45.

16. Batistao DWDF, Gontijo-Filho P, Conceiçao N, Oliveira AGD, Ribas RM. Risk factors for vancomycin-resistant enterococci colonisation in critically ill patients. Mem Inst Oswaldo Cruz 2012; 107(1): 57-63.

17. Peel T, Cheng AC, Spelman T, Huysmans M, Spelman D. Differing risk factors for vancomycin-resistant and vancomycinsensitive enterococcal bacteraemia. Clin Microbiol Infect 2012; 18(4): 388-94.

18. Akturk H, Sutcu M, Somer A, Karaman S, Acar M, Unuvar A, et. al. Results of Four-Year Rectal Vancomycin-Resistant Enterococci Surveillance in a Pediatric Hematology-Oncology Ward: From Colonization to Infection. Turk J Haematol 2016; 33(3): 244-47.

19. Matar MJ, Safdar A, Rolston K. Relationship of colonization with vancomycin- resistant enterococci and risk of systemic infection in patients with cancer. Clin Infec Dis 2006; 42(10): 1506-1507.

20. Rolston KV, Jiang Y, Matar M. VRE fecal colonization/infection in cancer patients. Bone Marrow Transplant 2007; 39(9): 567-68.

21. Agegne M, Abera B, Derbie A, Yismaw G, Shiferaw MB. Magnitude of vancomycin-resistant enterococci (VRE) colonization among hiv-infected patients attending art clinic in west amhara government hospitals. Int J Microbiol 2018; 2018(1): 7510157-60.

22. Sakka V, Tsiodras S, Galani L, Antoniadou A, Souli M, Galani I, et.al. Risk-factors and predictors of mortality in patients colonised with vancomycin-resistant enterococci. Clin Microbiol Infect 2008; 14(1): 14-21.

23. Kara A, Devrim J, Bayram N, Katipoglu N, Kiran E, Oruc Y, et. al. Risk of vancomycin resistant enterococci bloodstream infection among patients colonized with vancomycin resistant enterococci. Braz J Infect Dis 2015; 19(1): 58-61.

24. Melese A, Genet C, Andualem T. Prevalence of vancomycin resistant enterococci (VRE) in ethiopia: a systematic review and meta-analysis. BMC Infect Dis 2020; 20(): 124-30. 